\title{
Representations of Place in the Human Brain
}

\author{
Amy Lobben ${ }^{a^{*}}$, Megan Lawrence ${ }^{\mathrm{b}}$, P. William Limpisathian ${ }^{\mathrm{a}}$ \\ ${ }^{a}$ University of Oregon,lobben@uoregon.edu,billl@uoregon.edu \\ ${ }^{b}$ Microsoft,melawre@microsoft.com \\ * Corresponding author
}

Keywords: Parahippocampal Place Area, PPA, Neurogeography, fMRI

\begin{abstract}
:
Cartographers have been representing places and scenes for thousands of years. We know that there is not one single way to represent place. We can use a reference map, thematic map (not to mention all the different types), large scale, small scale, oblique and overhead remotely sensed image, hand-drawn cartoon maps, street view photographs, animated maps, and digital maps. We also know that the methods and then the resulting representations can be differentiated cartographically using established criteria. But, are these methods all equally effective in conveying a sense of place?
\end{abstract}

We measure "effectiveness" by comparing activation differences in the parahippocampal place area when viewing different representations of place. The parahippocampal place area (PPA) is a region in the human brain that allows humans to recognize and characterize a place (or a representation of it) (Weiner et al., in press). The PPA is a functionally, as opposed to an anatomically, defined region. It overlaps several anatomical regions, including the parahippocampal cortex, the lingual gyrus, the collateral sulcus, and the fusiform gyrus (Figure 1) (Epstein, 2014). The place recognition function of the PPA has been well-documented (Weiner et al., in press; Epstein 2014, 2008; Baldassano et al. 2013; Aguirre et al., 1998; Epstein and Kanwisher, 1998), and we now know that this region is what allows humans to differentiate between a place and other objects such as faces, chairs, or apples.

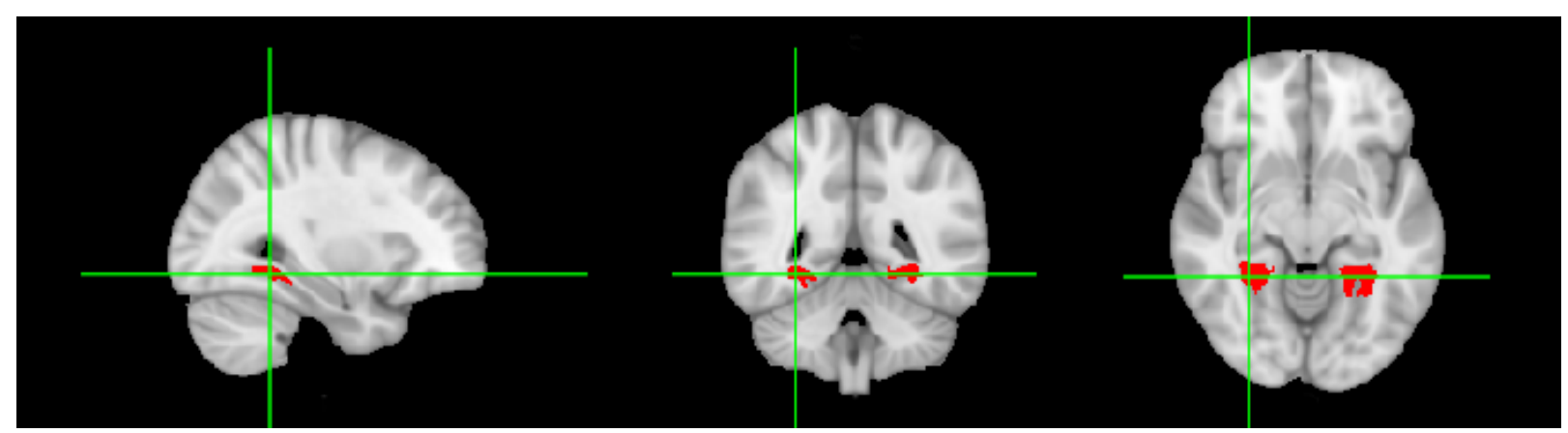

Figure 1. The PPA as represented by Baldassano's mask.

In this study, we measured the effect of cartographic representation on the human brain's recognition of "place" by comparing the activation differences in the PPA. We compared four types of place representation: a street-view photograph of an urban environment, a drawn schematic similar to a subway-style map, a Google Maps street map, and a Google Maps satellite view. (Figure 2). The Google Maps images were used because they are common cartographic representations, and thus are likely representative of a general "map" condition.

We utilized a block design fMRI experiment, in which each graphic type was displayed eight times in rapid succession before a rest period. The participants were first shown a blank screen for one second, followed by eight iterations of a task graphic, each for 3 seconds, followed by a twelve second rest period. For each task graphic, the participants were asked to respond using a button if the image shown was the same as the one immediately previous. This 1-back task helps ensure that the participants continue to pay attention during the block. This block was repeated six times during one full scan, and the scan was repeated for each experimental condition. After each experimental scan was completed, an anatomical scan was run, followed by a repeat of the experimental scan. 

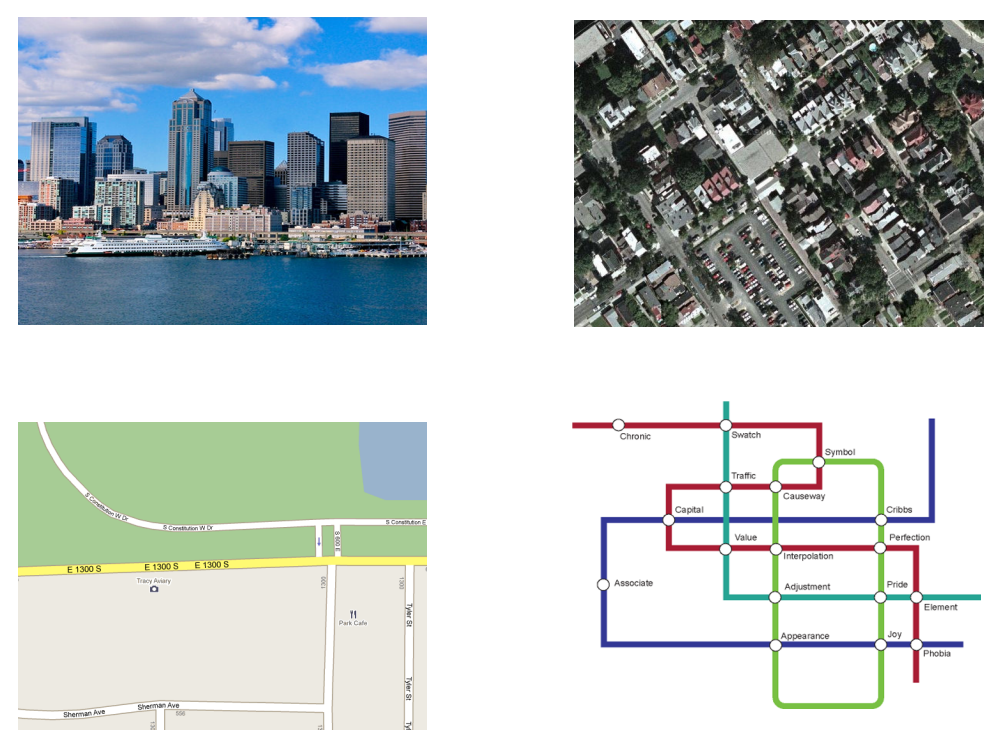

Figure 2. Examples of the Stimuli Categories.
Ten participants, four women and six men, with normal or corrected-to-normal vision from the University of Oregon community, were recruited for the study. The mean age of participants was 29.5 with a standard deviation of 6.98 and a range of 20 to 41 . Informed consent was obtained for each participant according to procedures approved by the Institutional Review Board at the University of Oregon, and each participant was paid for their participation.

Once the images were collected from the scanner, a series of pre-processing steps were required. First, the Brain Extraction Tool (BET) in the FMRIB Software Library (FSL) was used to isolate the brain from the skull in the functional images. We applied the Motion Correction using FMRIB's Linear Image Registration Tool (MCFLIRT) from the fMRI Expert Analysis Tool (FEAT) in FSL in order to identify any motion correction parameters that would indicate severe motion. Confound regressors were generated for each spike's time point. The functional images were realigned using MCFLIRT, and then spatially smoothed.

The predicted neurological response was then entered into a general linear model (GLM) along with the motion correction parameters and motion artifact confound variables. The GLM generated parameter estimates for each voxel in the time series, and these estimates were then contrasted against a control image (a graphic of scrambled pixels from each of the task stimuli). This contrast utilized the subtractive method, which subtracts activation present in the control condition from the activation in the experimental condition. This method thereby isolates activation unique to the task, and removes activation that may be caused by the presentation, such as pressing a button to respond to the question. This process resulted in a statistical map of task related brain activations contrasted against control related brain activations. The statistical map consists of $t$ scores for the contrast for each voxel, which were then thresholded to a minimum $p$-value of 0.05 and a minimum t-statistic of 2.3 (corrected for multiple comparisons using Gaussian Random Field theory). These statistical maps were then aligned to the Montreal Neurological Institute (MNI) 152 T1 2 millimeter brain. These individual results were then run through a higher-level analysis where the individual contrasts were aggregated across subjects using a group fixed effects analysis. The activation was then masked using areas defined by a functional PPA mask developed by Baldassano (2013) and shown in Figure 1.

The results of the contrasts between the activation in the PPA for experimental conditions and the scrambled graphic control revealed differential activation in the PPA when viewing the photograph, map, satellite conditions, and schematic map (respectively). The outcomes support what cartographers have long known - the process of selection and generalization necessarily transform the real world into a partial and restricted representation of space. The human mind agrees. While our brain still recognizes a map as a place, the cartographic transformation process seems to result in maps being perceived as less "place-like" when compared to photographs of the real world.

Aguirre, G.K., Zarahn, E., D'Esposito, M., 1998. An area within human ventral cortex sensitive to "building" stimuli: evidence and implications. Neuron 21, 373-383.

Baldassano, C., Beck, D.M., Fei-Fei, L., 2013. Differential connectivity within the parahippocampal place area. Neuroimage 75, 228-237.

Epstein, R., 2008. Parahippocampal and retrosplenial contributions to human spatial navigation. Trends in Cognitive Science. 12 (10), 388-396.

Epstein, R., 2014. Neural systems for visual scene recognition. In: Bar, M., Keveraga, K. K.S. (Eds.), Scene Vision. MIT Press, Cambridge MA, 105-134.

Epstein, R., Kanwisher, N., 1998. A cortical representation of the local visual environment. Nature 392, $598-601$.

Smith S. M., M. Jenkinson, M. W. Woolrich, C. F. Beckmann, T. E. J. Behrens, H. Johansen-Berg, P. R. Bannister, M. De Luca, I. Drobnjak, D. E. Flitney, R. Niazy, J. Saunders, J. Vickers, Y. Zhang, N. De Stefano, J. M. Brady, and P. M. Matthews. 2004. Advances in functional and structural MR image analysis and implementation as FSL. NeuroImage 23 (21): 208-219.

Weinera, K.S , M. A. Barnetta, N. Witthofta, G. Golaraia, A. Stigliania, K. N. Kayb , J. Gomezc , V. S. Natua, K. Amuntsd, K. Zillesd, K. Grill-Spector (in press). Defining the most probable location of the parahippocampal place area using cortex-based alignment and cross-validation. NeuroImage $\mathrm{xxx}(\mathrm{xxxx}) \mathrm{xxx}-\mathrm{xxx}$ 